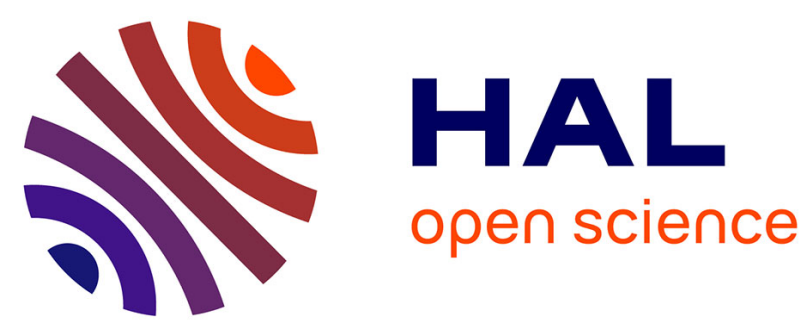

\title{
Complex emergent properties in synchronized neuronal oscillations
}

\author{
Nathalie Corson, Moulay Aziz-Alaoui
}

\section{To cite this version:}

Nathalie Corson, Moulay Aziz-Alaoui. Complex emergent properties in synchronized neuronal oscillations. Springer. From System Complexity to Emergent Properties, Springer - Understanding Complex Systems Series, p. 243, 2009, Understanding Complex Systems Series. hal-00952594

\section{HAL Id: hal-00952594 \\ https://hal.science/hal-00952594}

Submitted on 27 Feb 2014

HAL is a multi-disciplinary open access archive for the deposit and dissemination of scientific research documents, whether they are published or not. The documents may come from teaching and research institutions in France or abroad, or from public or private research centers.
L'archive ouverte pluridisciplinaire HAL, est destinée au dépôt et à la diffusion de documents scientifiques de niveau recherche, publiés ou non, émanant des établissements d'enseignement et de recherche français ou étrangers, des laboratoires publics ou privés. 


\title{
Complex emergent properties in synchronized neuronal oscillations
}

\author{
Nathalie Corson and M.A. Aziz-Alaoui \\ Laboratoire de Mathématiques Appliquées du Havre \\ 25 rue Philippe Lebon \\ 76600 Le Havre, France \\ nathalie.corson@univ-lehavre.fr, aziz.alaoui@univ-lehavre.fr
}

Summary. This work adresses the dynamics and complexity of the HindmarshRose neuronal mathematical model. The general aim is the study of the asymptotic behaviour of neuron networks. In this paper, the analysis of these networks uses the synchronization theory via connections between neurons which give rise to emergent properties and self-organization. Our results lead to a classical law which describes many natural or artificial self-organized complex systems. This has been performed using numerical tools.

Key words : Hindmarsh-Rose model, synchronization, complexity, emergent properties.

\section{Introduction}

Also called nerve cells, neurons are the most important cells of the nervous system. The Hindmarsh-Rose model ( $H R$ hereafter), see $[7,8]$, describes the dynamics of the membrane potential in the axon of a neuron with a three dimensional system of nonlinear first order differential equations which read as,

$$
\left\{\begin{array}{l}
\dot{x}=y+a x^{2}-x^{3}-z+I \\
\dot{y}=1-d x^{2}-y \\
\dot{z}=\epsilon\left(b\left(x-x_{c}\right)-z\right)
\end{array}\right.
$$

While $x$ describes the membrane potential, $y$ describes the exchange of ions accross the neuron membrane through fast ionic channels and $z$ the exchange of ions through slow ionic channels.

$I$ is the applied current while $\epsilon$ is a recovery variable, which is very small. This last parameter controls the slow motion of the neuron activity. Parameters $a, b, d$ are constants experimentally determined and $x_{c}$ is the equilibrium 
$x$-coordinate of the two dimensional Hindmarsh-Rose system composed of the first two equations of (1) with $z=0$ and $I=0$.

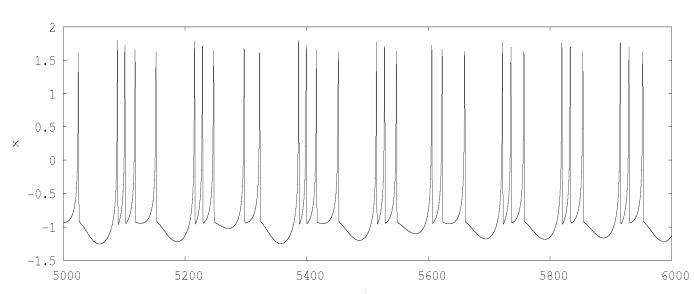

(a)

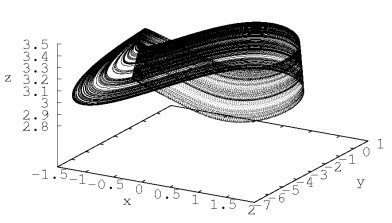

(b)

Fig. 1. System (1) for $a=3, \quad b=4, \quad d=5, \quad x_{c}=-\frac{1}{2}(1+\sqrt{5}), I=3.25$ and $\epsilon=0.001$. (a) Time series $(t, x)$, (b) $(x, y, z)$ view.

In this paper, we mainly study synchronization motions of coupled $H R$ systems which can be chaotic for some parameters choices. See for example $[5,6,10,16]$, or $[9,14,15,17]$ in which the behaviour bursting, spiking chaos or adding bifurcation are studied. For the reader convenience, let us recall that synchronization is a phenomenon characteristic of many processes in natural systems and nonlinear science. It has remained an objective of intensive research and is today considered as one of the basic nonlinear phenomena studied in mathematics, physics, engineering or life science. Several different regimes of synchronization have been investigated by many authors, see for example $[1,4,11,12,13]$.

This word has a greek root, syn $=$ common and chronos $=$ time, which means to share common time or to occur at the same time, that is correlation or agreement in time of different processes.

Thus, synchronization of two dynamical systems generally means that one system somehow follows the motion of another. A lot of research has been carried out and, as a result, showed that even chaotic systems could synchronize when they are coupled. Many researchers have discussed the theory, the design or applications of synchronized motion in coupled chaotic systems. A broad variety of applications have emerged, for example to increase the power of lasers, to synchronize the output of electronic circuits, to control oscillations in chemical reactions or to encode electronic messages for secure communications. Here are some synchronization regimes,

- Identical (or complete) Synchronization, which is defined as the coincidence of states of interacting systems,

- Generalized Synchronization, which extends the identical synchronization phenomenon and implies the presence of some functional relation between two coupled systems; if this relationship is the identity we recover the identical synchronization, 
- Phase Synchronization, which means driving of phases of chaotic oscillators, whereas their amplitudes remain uncorrelated,

- Lag Synchronization, which appears as a coincidence of shifted-in-time states of two systems.

Hereafter, for all numerical experiments, we use $H R$ system with the following coordinates changes, see [3], $y=1-y, z=1+I+z, d=a+\alpha, c=-1-I-b x_{c}$. From this changes of coordinates, we obtain,

$$
\left\{\begin{array}{l}
\dot{x}=a x^{2}-x^{3}-y-z \\
\dot{y}=(a+\alpha) x^{2}-y \\
\dot{z}=\epsilon(b x+c-z)
\end{array}\right.
$$

Let us consider a network composed by $n H R$ neurons. These neurons are coupled by their first variable $x_{i}$. A model of this network is given by,

$$
\left\{\begin{array}{l}
\dot{x_{i}}=a x_{i}^{2}-x_{i}^{3}+y_{i}-z_{i}-h\left(x_{i}, x_{j}\right), \quad i \neq j, \quad i=1, \ldots, n, \quad j=1, \ldots, n \\
\dot{y_{i}}=(a+\alpha) x_{i}^{2}-y_{i} \\
\dot{z_{i}}=\epsilon\left(b x_{i}+c-z_{i}\right)
\end{array}\right.
$$

In the case of a network of $n$ neurons linearly coupled, the coupling function $h$ is chosen as,

$$
h\left(x_{i}, x_{j}\right)=k_{n} \sum_{j=1}^{n} c_{i j} \Gamma\left(x_{j}\right)
$$

in which the synaptic coupling $\Gamma$ is a linear function, $\Gamma\left(x_{j}\right)=x_{i}-x_{j}$, for $i=1,2, \ldots, n$. Parameter $k_{n}$ represents the coupling strength and $C_{n}=\left(c_{i j}\right)$ is the $n \times n$ connectivity matrix,

$$
\left\{\begin{array}{l}
c_{i j}=1 \text { if } \mathrm{i} \text { and } \mathrm{j} \text { are connected, } i=1, \ldots n, j=1, \ldots n, i \neq j \\
c_{i j}=0 \text { if } \mathrm{i} \text { and } \mathrm{j} \text { are not connected. }
\end{array}\right.
$$

This matrix $C_{n}$ can be symmetric or not, so that unidirectional or bidirectional coupling are possible.

In the case of a network of $n$ neurons nonlinearly coupled, the coupling function $h$ is given by [3] and reads as,

$$
h\left(x_{i}, x_{j}\right)=\left(x_{i}-V\right) k_{n} \sum_{j=1}^{n} c_{i j} \Gamma\left(x_{j}\right)
$$

in which the synaptic coupling $\Gamma$ is a nonlinear function with a threshold as given,

$$
\Gamma\left(x_{j}\right)=\frac{1}{1+\exp \left(-\lambda\left(x_{j}-\Theta\right)\right)}
$$


where $\Theta$ is the threshold reached by every action potential for a neuron and $\lambda$ is defined in [3].

As in the case of linear coupling, parameter $k_{n}$ corresponds to the synaptic coupling strength. Parameter $V$ is the reversal potential and must be larger than $x_{i}(t)$ for all $i$ and all $t$ since synapses are supposed excitatory.

We have chosen the situation in which each neuron has the same number of inputs from other neurons. Indeed, according to [3], it is a necessary condition for the synchronous solution to exist. Therefore, the synaptic connections we use have to be bidirectional. The $C_{n}$ matrix should be symmetric and in our case, $C_{n}=1_{n}-I d_{n}$ where $1_{n}=(1)_{n n}$ and $I d_{n}=(I d)_{n n}$ is the identical matrix.

The following parameters are fixed as follows throughout this paper,

$$
\begin{gathered}
a=2.8, \alpha=1.6, c=5, b=9, \epsilon=0.001 \\
V=2, \lambda=10, \Theta=-0.25
\end{gathered}
$$

Interactions between nerve cells are possible thanks to synapses. The word synapse has a greek root, syn = common and haptein $=$ to touch, which means connection. This is the functional contact part which exists between two neurons or between a neuron and another cell (muscular cell, sensorial receptor, ...). It operates the conversion of an action potential fired by the presynaptic neuron into a signal in the postsynaptic cell. Usually, two different types of synapses are discerned, which are,

- the chemical synapse, widely majoritary, which needs some neurotransmitters to transmit information,

- the electrical synapse, in which the signal is transmitted electrically through gap-junctions.

The size of the synaptic cleft is characteristic of one or the other kind of synapse. In the case of electrical synapses, this synaptic cleft is about two nanometers, while it can reach from ten to forty nanometers in the case of chemical synapses.

This paper is organized as follows. In the next section, the coupling function between mathematical neurons is a linear function in order to represent electrical synaptic connections. In this section, we firstly assume that neurons are identical and then, we assume that they are slightly different. In section 3 , the same steps are followed with a nonlinear function in order to study chemical synaptic connections. 


\section{Property emerging from synchronization with a linear coupling}

In this paper, the asymptotic behaviour is studied only by numerical analysis in various regions of the parameter space commonly used in the litterature. We have done the numerical computations very accurately in double precision and for different variations of parameters. In order to obtain reliable numerical results, the step size has been chosen to be equal to $10^{-4}$ and the first $10^{6}$ steps are discarded to avoid the transient regime.

\subsection{Coupling identical $H R$ neurons}

The first step is to consider the asymptotic behaviour of two neurons lineraly coupled. Therefore, we numerically study system (3) with coupling function (4) for $n=2$. The connectivity matrix $C_{2}$ and the graph of the network are given in figure 2 .

$$
C_{2}=1_{2}-I d_{2}=\left[\begin{array}{ll}
0 & 1 \\
1 & 0
\end{array}\right]
$$

Fig. 2. Bidirectional connection scheme for two neurons and the connectivity matrix $\mathrm{C}_{2}$.

This gives the six-dimensional system representing two bidirectionaly coupled $H R$ neurons given in (9),

$$
\left\{\begin{array}{l}
\dot{x_{1}}=a x_{1}^{2}-x_{1}^{3}+y_{1}-z_{1}-k_{2}\left(x_{1}-x_{2}\right) \\
\dot{y_{1}}=(a+\alpha) x_{1}^{2}-y_{1} \\
\dot{z_{1}}=\epsilon\left(b x_{1}+c-z_{1}\right) \\
\dot{x_{2}}=a x_{2}^{2}-x_{2}^{3}+y_{2}-z_{2}-k_{2}\left(x_{2}-x_{1}\right) \\
\dot{y_{2}}=(a+\alpha) x_{2}^{2}-y_{2} \\
\dot{z_{2}}=\epsilon\left(b x_{2}+c-z_{2}\right)
\end{array}\right.
$$

Figure 3 shows the evolution of the $\left(x_{1}, x_{2}\right)$ phase portrait of system (9) while making the coupling strength $k_{2}$ increase after a certain period of time. For low values of this coupling strength, the behaviour of $x_{1}$ is really different from the one of $x_{2}$, whereas when this coupling strength is large enough, $x_{1}$ and $x_{2}$ behaviours become synchronous. 


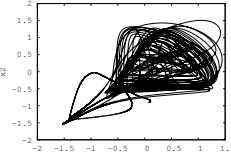

(a)

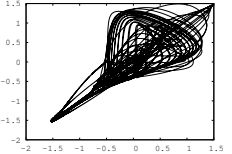

(b)

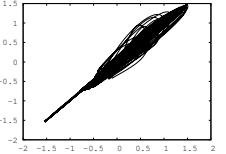

(c)

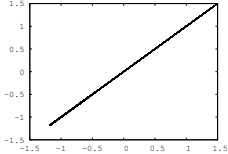

(d)

Fig. 3. Phases portraits for system (9) showing $x_{2}$ according to $x_{1}$ for the coupling strength (a) $k_{2}=0.2$, (b) $k_{2}=0.25$, (c) $k_{2}=0.36$, (d) $k_{2}=0.376$. For two neurons linearly coupled, the synchronization threshold for the coupling strength is about $k_{2}=0.376$.

We observe that synchronization motion between the first variable $x_{1}$ of one neuron and the first variable $x_{2}$ of the other, i.e : $x_{1}=f\left(x_{2}\right)$ (here, $f(x)=I d(x)$ ), appears for a coupling strength $k_{2} \geq 0.376$. For every coupling strength larger that this value of $k_{2}, x_{1}$ and $x_{2}$ remain synchronous. This phenomenon is also observed for variables $y$ and $z$, for the same values of parameters. Therefore, this synchronization is total.

In the case of three neurons, the connectivity matric $C_{3}$ and the graph of the network are given in figure 4.

$$
C_{3}=1_{3}-I d_{3}=\left[\begin{array}{lll}
0 & 1 & 1 \\
1 & 0 & 1 \\
1 & 1 & 0
\end{array}\right]
$$

Fig. 4. Bidirectional connection scheme for three neurons and the connectivity matrix $C_{3}$.

System (3) with coupling function (4) for $n=3$ reads as,

$$
\left\{\begin{array}{l}
\dot{x_{1}}=a x_{1}^{2}-x_{1}^{3}+y_{1}-z_{1}-k_{3}\left(x_{1}-x_{2}\right)-k_{3}\left(x_{1}-x_{3}\right) \\
\dot{y_{1}}=(a+\alpha) x_{1}^{2}-y_{1} \\
\dot{z_{1}}=\epsilon\left(b x_{1}+c-z_{1}\right) \\
\dot{x_{2}}=a x_{2}^{2}-x_{2}^{3}+y_{2}-z_{2}-k_{3}\left(x_{2}-x_{1}\right)-k_{3}\left(x_{2}-x_{3}\right) \\
\dot{y_{2}}=(a+\alpha) x_{2}^{2}-y_{2} \\
\dot{z_{2}}=\epsilon\left(b x_{2}+c-z_{2}\right) \\
\dot{x_{3}}=a x_{3}^{2}-x_{3}^{3}+y_{3}-z_{3}-k_{3}\left(x_{3}-x_{1}\right)-k_{3}\left(x_{3}-x_{2}\right) \\
\dot{y_{3}}=(a+\alpha) x_{3}^{2}-y_{3} \\
\dot{z_{3}}=\epsilon\left(b x_{3}+c-z_{3}\right)
\end{array}\right.
$$

As shown in the case of two neurons, figure 5 shows the evolution of $x_{1}$ according to $x_{2}$ for system (10) while making the coupling strength increase. 
For low values of this coupling strength, the behaviour of $x_{1}$ is really different from the one of $x_{2}$, whereas when this coupling strength is large enough, $x_{1}$ and $x_{2}$ behaviours become identical.

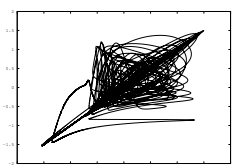

(a)

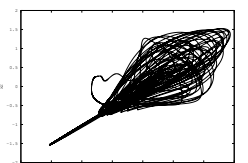

(b)

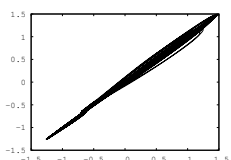

(c)

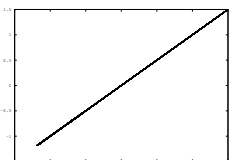

(d)

Fig. 5. Phases portraits for system (10) showing $x_{2}$ according to $x_{1}$ for the coupling strength (a) $k_{3}=0.1$, (b) $k_{3}=0.2$, (c) $k_{3}=0.24$, (d) $k_{3}=0.255$. For three linearly coupled neurons, the synchronization threshold of the coupling strength is around $k_{3}=0.255$.

If figure 5 only shows the $\left(x_{1}, x_{2}\right)$ view of the phase portraits of system (10), a similar phenomenon is observed for between $x_{1}$ and $x_{3}$. Therefore, the minimum coupling strength $k_{3}$ to exhibit synchronization between the first variables $x_{1}, x_{2}$ and $x_{3}$ of three $H R$ neurons is about $k_{3}=0.255$. Once again, we observe also this phenomenon for the variables $y$ and $z$ for the same numerical values of parameters. This is a synchronization threshold.

There are different possible ways for coupling four, five or more neurons. However, as we already said, we consider the case in which each neuron is connected to all the others. Therefore, there is a unique possible connection scheme.

Here, we consider system (3) with coupling function (4) for $n=4, n=5$, $n=6, n=7, n=8$. Figures $6,8,10,12$ and 14 show the connection graphs of the network and the connectivity matrix $C_{n}$ respectively in the case of 4 , 5, 6, 7 and 8 neurons coupled all together.

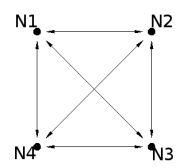

Fig. 6. Bidirectional connection scheme for four neurons. The connectivity matrix $C_{4}$ is $C_{4}=1_{4}-I d_{4}$.

Figures $7,9,11,13,15$ show the evolution of the $\left(x_{1}, x_{2}\right)$ phase portrait while making the coupling strength increase in the case of $4,5,6,7$ and 8 neurons coupled all together using the coupling function given in (4). These phase portraits are plotted after a period of time. These figures show $x_{2}$ according 
to $x_{1}$, but the same motion is observed comparing the behaviour of the first variable of the other corresponding variables.

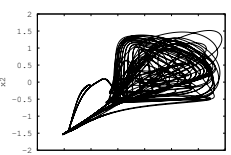

(a)

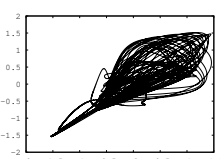

(b)

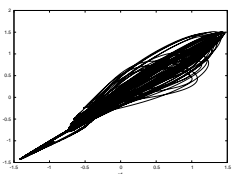

(c)

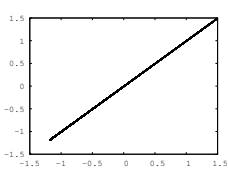

(d)

Fig. 7. $x_{2}$ according to $x_{1}$ when four neurons are connected for the coupling strength (a) $k_{4}=0.1$, (b) $k_{4}=0.15$, (c) $k_{4}=0.18$, (d) $k_{4}=0.191$. Minimum coupling strength to observe synchronization between variables $x_{i}$ and $x_{j}, y_{i}$ and $y_{j}, z_{i}$ and $z_{j}(i=1, \ldots, 4, \quad j=1, \ldots, 4, \quad i \neq j)$ when coupling four neurons is $k_{4}=0.191$.

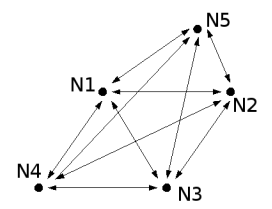

Fig. 8. Coupling five neurons. The connectivity matric $C_{5}$ is $C_{5}=1_{5}-I d_{5}$.

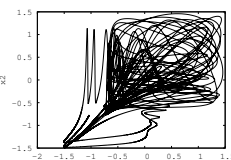

(a)

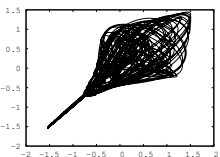

(b)

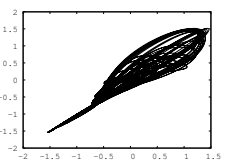

(c)

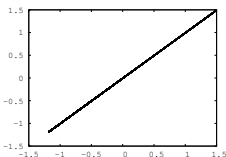

(d)

Fig. 9. $x_{2}$ according to $x_{1}$ when five neurons are connected for the coupling strength (a) $k_{5}=0.05$, (b) $k_{5}=0.11$, (c) $k_{5}=0.14$, (d) $k_{5}=0.152$. Minimum coupling strength to observe synchronization between variables $x_{i}$ and $x_{j}, y_{i}$ and $y_{j}, z_{i}$ and $z_{j}(i=1, \ldots, 5, \quad j=1, \ldots, 5, \quad i \neq j)$ when coupling five neurons is $k_{5}=0.152$.

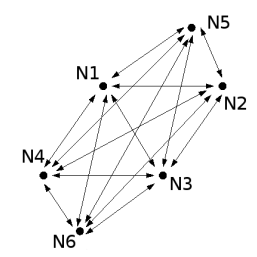

Fig. 10. Coupling six neurons. The connectivity matrix $C_{6}$ is $C_{6}=1_{6}-I d_{6}$. 


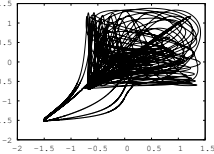

(a)

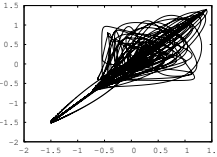

(b)

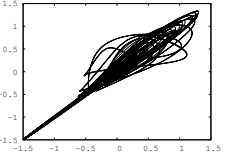

(c)

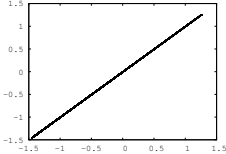

(d)

Fig. 11. $x_{2}$ according to $x_{1}$ when six neurons are connected for the coupling strength (a) $k_{6}=0.03$, (b) $k_{6}=0.05$, (c) $k_{6}=0.1$, (d) $k_{6}=0.122$. Minimum coupling strength to observe synchronization between variables $x_{i}$ and $x_{j}, y_{i}$ and $y_{j}, z_{i}$ and $z_{j}(i=1, \ldots, 6, \quad j=1, \ldots, 6, \quad i \neq j)$ when coupling six neurons is $k_{6}=0.122$.

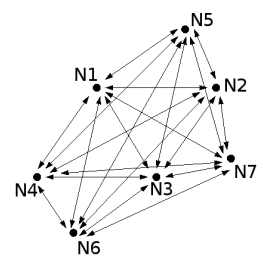

Fig. 12. Coupling seven neurons. The connectivity matrix $C_{7}$ is $C_{7}=1_{7}-I d_{7}$.

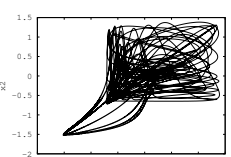

(a)

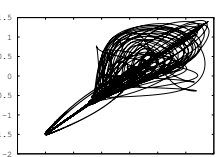

(b)

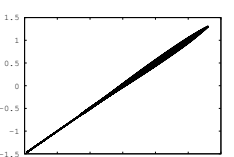

(c)

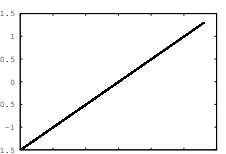

(d)

Fig. 13. $x_{2}$ according to $x_{1}$ when seven neurons are connected for the coupling strength (a) $k_{7}=0.025$, (b) $k_{7}=0.05$, (c) $k_{7}=0.1$, (d) $k_{7}=0.104$. Minimum coupling strength to observe synchronization between variables $x_{i}$ and $x_{j}, y_{i}$ and $y_{j}, z_{i}$ and $z_{j}(i=1, \ldots, 7, \quad j=1, \ldots, 7, \quad i \neq j)$ when coupling seven neurons is $k_{7}=0.104$.

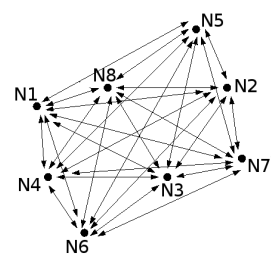

Fig. 14. Coupling eight neurons. The connectivity matrix $C_{8}$ is $C_{8}=1_{8}-I d_{8}$. 


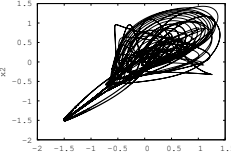

(a)

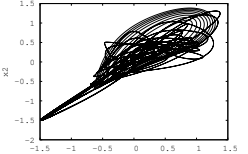

(b)

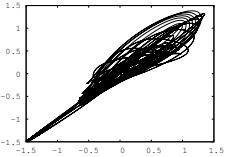

(c)

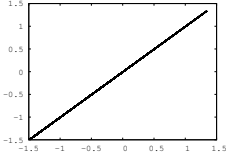

(d)

Fig. 15. $x_{2}$ according to $x_{1}$ when eight neurons are connected for the coupling strength (a) $k_{8}=0.05$, (b) $k_{8}=0.07$, (c) $k_{8}=0.08$, (d) $k_{8}=0.088$. Minimum coupling strength to observe synchronization between variables $x_{i}$ and $x_{j}, y_{i}$ and $y_{j}, z_{i}$ and $z_{j}(i=1, \ldots, 8, \quad j=1, \ldots, 8, \quad i \neq j)$ when coupling eight neurons is $k_{8}=0.088$.

Synchronization thresholds of the coupling strength we obtained are summarized in table 1.

\begin{tabular}{|c|c|c|c|c|c|c|c|}
\hline$n$ & 2 & 3 & 4 & 5 & 6 & 7 & 8 \\
\hline$k_{n}$ & 0.376 & 0.255 & 0.191 & 0.152 & 0.122 & 0.104 & 0.088 \\
\hline$K_{n}$ & & 0.303 & 0.202 & 0.151 & 0.121 & 0.101 & 0.086 \\
\hline
\end{tabular}

Table 1. Tabular summarizing minimal coupling strength $k_{n}$ to observe synchronous motion of $n$ neurons, with $n=2, \ldots, 8$ when coupling identical $H R$ neurons using the linear function (4). $K_{n}$ is the theoretical value of coupling strength obtained with the heuristic law given in (11).

In this tabular, the first column shows the number $n$ of neurons in the network. The second column shows the numerical results we obtained while the last column shows the theoretical results obtained thanks to the heuristic law we observed,

$$
K_{n}=\frac{k_{2}}{0.62 *(n-1)}
$$

Figure 16 (a) uses table 1 to show $k_{n}$ versus $n$ (dots) and $K_{n}$ versus $n$ (solid lines). Figure 16 (b) shows the curve obtained plotting $\log \left(K_{n}\right)$ according to $\log (n)$. 


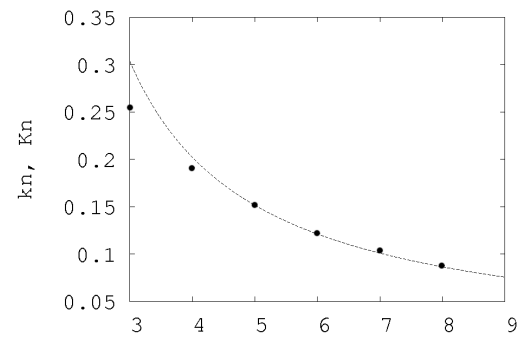

(a)

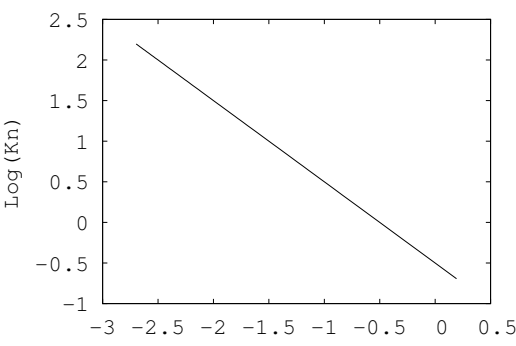

(b)

Fig. 16. (a) Minimum coupling strength $k_{n}$ to observe a synchronous behaviour of $n$ neurons, according to the number of neurons in the network (dots) plotted together with $K_{n}=k_{2} /(0.62(n-1))$ (solid line). (b) $\log \left(K_{n}\right)$ according to $\log (n)$.

The curve of figure 16 (b) corresponds to a classical law (for $n$ large enough) which can describe many self-organized complex systems, like earthquakes (for 1000 earthquakes of magnitude 4 on Richter scale, there are only 100 of magnitude 5 and 10 of magnitude 6), linguistics (for 1000 occurrences of "the" in an english text, there are only 100 occurrences of "I" and 10 of "say"), urban systems (big cities are rare and small ones are frequent in an exponential way).

\subsection{Coupling non-identical $H R$ neurons}

In this subsection, the same coupling function (4) is used to study the asymptotic behaviour of networks composed of $H R$ neuron which parameters are slightly different from one another. Indeed, in nature, it is not realistic to assume that a neuron network is composed of exactly identical neurons. We then make all parameters vary slightly from one neuron to another. Let $p_{i}$ be a generic way of writing every parameters of neuron $i$ (i.e. $a_{i}, \alpha_{i}, b_{i}, c_{i}$ ). The variation of parameters from one neuron to another is defined as in (12). It has been choosen around $e=10^{-4}$,

$$
\left\{\begin{array}{l}
p_{i}=p_{1}+\left(1+\frac{i}{2} 10^{-1}\right) \times e, \quad i=2 j, \quad j=1,2,3,4 \\
p_{i}=p_{1}+\left(1-\frac{i-1}{2} 10^{-1}\right) \times e, i=2 j+1, j=1,2,3
\end{array}\right.
$$

With these variations of parameters from a neuron to another, we obtain the following tabular (Table 2) of minimal coupling strength to obtain synchronization, and figure 17 showing a similar law as given in figure 16 . 


\begin{tabular}{|c|c|c|c|c|c|c|c|}
\hline$n$ & 2 & 3 & 4 & 5 & 6 & 7 & 8 \\
\hline$k_{n}$ & 0.376 & 0.252 & 0.192 & 0.152 & 0.127 & 0.11 & 0.092 \\
\hline$K_{n}$ & & 0.303 & 0.202 & 0.151 & 0.121 & 0.101 & 0.086 \\
\hline
\end{tabular}

Table 2. Tabular summarizing minimal coupling strength $k_{n}$ to observe synchronous motion of $n$ neurons, with $n=2, \ldots, 8$ when coupling slightly distinct $H R$ neurons using linear function (4). The difference between neurons is around $10^{-4}$.

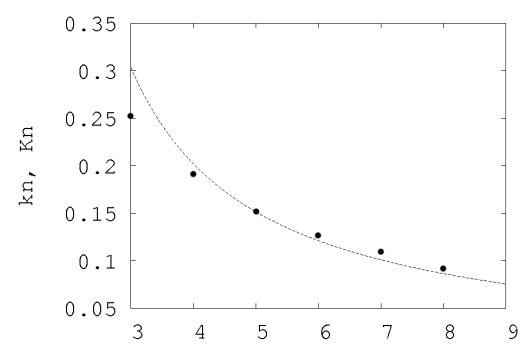

(a)

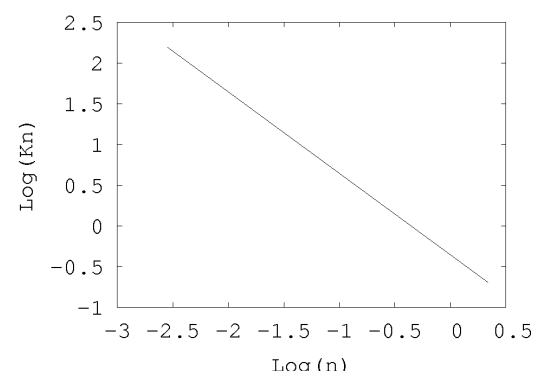

(b)

Fig. 17. (a) Minimum coupling strength $k_{n}$ to observe a synchronous behaviour of $n$ neurons according to the number of neurons in the network $n$ (dots) plotted together with $K_{n}=k_{2} /(0.62(n-1))$ (solid line). (b) $\log \left(K_{n}\right)$ according to $\log (n)$.

Then, in order to make the difference between neurons bigger, the variation is chosen as done in (12) with $e=10^{-3}$. With these variations of parameters from a neuron to another, we obtain the following tabular (Table 3 ) of synchronization thresholds, and figure 18 showing a similar law as previously presented.

\begin{tabular}{|c|c|c|c|c|c|c|c|}
\hline$n$ & 2 & 3 & 4 & 5 & 6 & 7 & 8 \\
\hline$k_{n}$ & 0.4 & 0.275 & 0.210 & 0.170 & 0.130 & 0.115 & 0.098 \\
\hline$K_{n}$ & & 0.351 & 0.234 & 0.175 & 0.140 & 0.117 & 0.100 \\
\hline
\end{tabular}

Table 3. Tabular summarizing minimal coupling strength to observe synchronous motion of $n$ neurons, with $n=2, \ldots, 8$ when coupling slightly distinct $H R$ neurons using linear function (4). The difference betwenn neurons is around $10^{-3}$. 


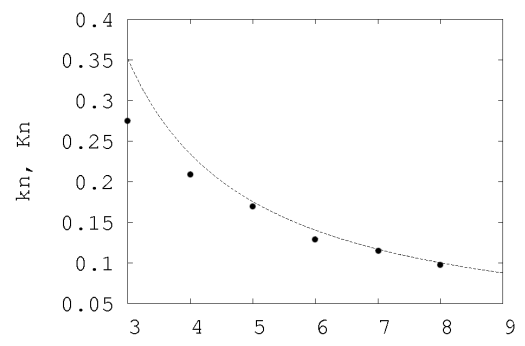

(a)

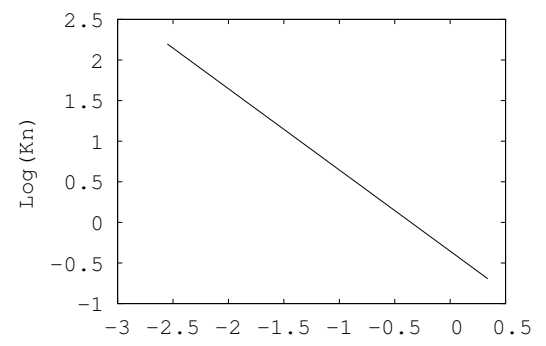

(b)

Fig. 18. (a) Minimum coupling strength $k_{n}$ to observe a synchronous behaviour of $n$ neurons according to the number of neurons in the network (dots) plotted together with $K_{n}=k_{2} /(0.57(n-1))$ (solid line). (b) $\log \left(K_{n}\right)$ according to $\log (n)$.

\section{Property emerging from synchronization with a nonlinear coupling}

\subsection{Coupling identical $H R$ neurons}

For $n=2$, system (3) with the sigmoïdal coupling function $h$ defined as in (5) and $\Gamma$ defined as in (6) reads as,

$$
\left\{\begin{array}{l}
\dot{x_{1}}=a x_{1}^{2}-x_{1}^{3}+y_{1}-z_{1}-\left(x_{1}-V\right) k_{2} \frac{1}{1+\exp \left(-\lambda\left(x_{2}-\Theta\right)\right)} \\
\dot{y_{1}}=(a+\alpha) x_{1}^{2}-y_{1} \\
\dot{z_{1}}=\epsilon\left(b x_{1}+c-z_{1}\right) \\
\dot{x_{2}}=a x_{2}^{2}-x_{2}^{3}+y_{2}-z_{2}-\left(x_{2}-V\right) k_{2} \frac{1}{1+\exp \left(-\lambda\left(x_{1}-\Theta\right)\right)} \\
\dot{y_{2}}=(a+\alpha) x_{2}^{2}-y_{2} \\
\dot{z_{2}}=\epsilon\left(b x_{2}+c-z_{2}\right)
\end{array}\right.
$$

For $n=3$, system (3) with the coupling function $h$ defined as in (5) and $\Gamma$ defined as in (6) reads as, 


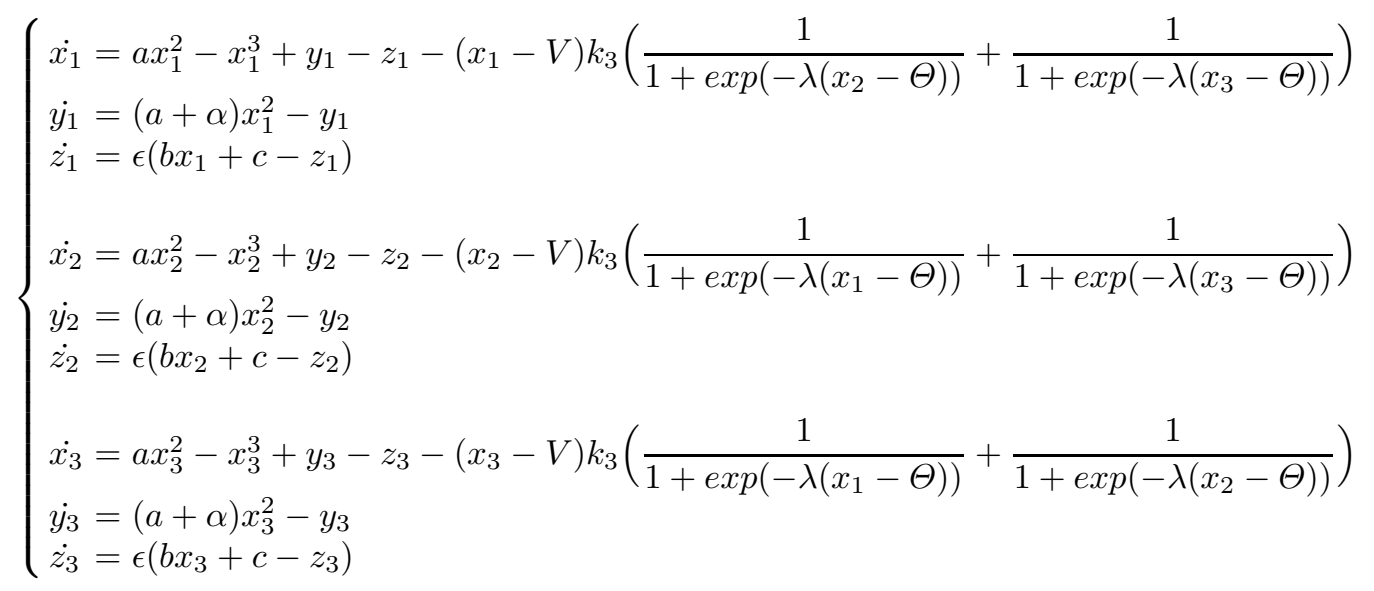

The same numerical experiments as in the previous section lead us to table 4 , in which theorical coupling strength $K_{n}$ given by the heuristic law (14) is also presented,

$$
K_{n}=\frac{k_{2}}{(n-1)}
$$

\begin{tabular}{|c|c|c|c|c|c|c|c|}
\hline$n$ & 2 & 3 & 4 & 5 & 6 & 7 & 8 \\
\hline$k_{n}$ & 1.26 & 0.63 & 0.5 & 0.3 & 0.24 & 0.21 & 0.17 \\
\hline$K_{n}$ & & 0.63 & 0.42 & 0.315 & 0.252 & 0.21 & 0.18 \\
\hline
\end{tabular}

Table 4. Tabular summarizing minimal coupling strength $k_{n}$ to observe synchronous motion of $n$ neurons, with $n=2, \ldots, 8$ when coupling identical $H R$ neurons using linear function (4) and the coupling strength obtained with the heuristic law given in (14).
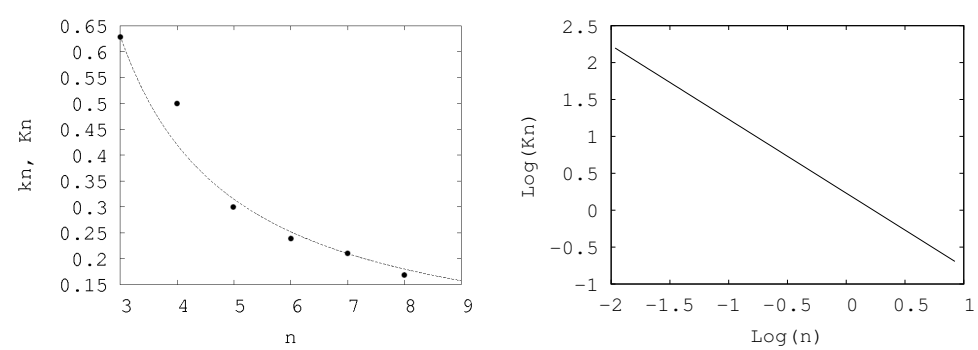

Fig. 19. (a) Minimum coupling strength $k_{n}$ to observe a synchronous behaviour of $n$ neurons according to the number of neurons in the network (dots) plotted together with $K_{n}=k_{2} /(n-1)$ (solid line). (b) $\log \left(K_{n}\right)$ according to $\log (n)$. 
As the previous numerical results show, the synchronization threshold follows the heuristic law,

$$
K_{n}=k_{2} /(n-1)
$$

This result has been first presented in [3].

\subsection{Coupling non-identical $H R$ neurons}

In this subsection, the coupling function (5), with $\Gamma$ defined as in (6), is used to study the asymptotic behaviour of networks composed of coupled $H R$ neurons, the parameters of which are slightly different from one another, as in subsection 2.2. The variation of parameters from one neuron to another is defined as in (12) with $e=10^{-4}$. With these different neurons, we obtain the following tabular (Table 5) which gives the synchronization thresholds from two to eight coupled neurons. The obtained results are similar to those we observed in the previous section. They show the same heuristic law given by equation (15). These results plotted figure 20.

\begin{tabular}{|c|c|c|c|c|c|c|c|}
\hline$n$ & 2 & 3 & 4 & 5 & 6 & 7 & 8 \\
\hline$k_{n}$ & 3.8 & 1.8 & 1.2 & 0.9 & 0.7 & 0.6 & 0.2 \\
\hline$K_{n}$ & & 1.9 & 1.26 & 0.95 & 0.76 & 0.63 & 0.54 \\
\hline
\end{tabular}

Table 5. Tabular summarizing minimal coupling strength $k_{n}$ to observe synchronous motion of $n$ neurons, with $n=2, \ldots, 8$ when coupling slightly distinct $H R$ neurons using the nonlinear function (5) $\Gamma$ defined as in (6). The third column shows the coupling strength obtained with the heuristic law given in (15).
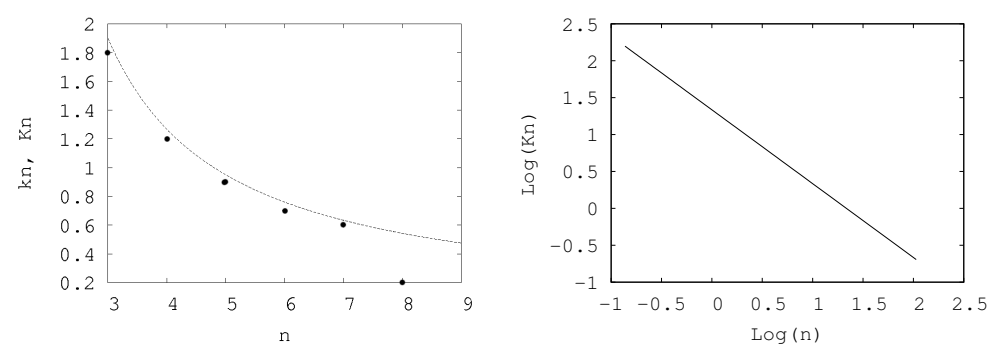

Fig. 20. (a) Minimum coupling strength $k_{n}$ to observe a synchronous behaviour of $n$ neurons according to the number of neurons in the network (dots) plotted together with $K_{n}=k_{2} /(n-1)$ (solid line). (b) $\log \left(K_{n}\right)$ according to $\log (n)$. 


\section{Conclusion}

Emergence and complexity refer to the appearance of higher-level properties and behaviours of a system that obviously comes from the collective dynamics of that system's components, see [2]. These properties are not directly deductable from the lower-level motion of that system. Emergent properties are properties of the 'whole' that are not possessed by any of the individual parts making up that whole. For example, an air molecule is not a cyclone, an isolated species does not form a food chain and an isolated neuron is not conscious : emergent behaviours are typically novel and unanticipated. Thus, if a synchronization phenomenon is exhibited in a neuron network, it may develop some kind of consciousness. This is an emergent property which comes from the collective dynamics of $n$ neurons. Moreover, as given in figure (19), as the number of neurons $n$ increases, the synchronization threshold $k_{n}$ decreases. Consciousness is more important when the number of neurons is larger?

\section{References}

1. M.A. Aziz-Alaoui (2006), Synchronization of chaos, Encyclopedia of mathematical physics, Elsevier vol.5, pp 213-226

2. M.A. Aziz-Alaoui (2006), Complex emergent properties and chaos (De) synchronization, in Emergent Properties in Natural and Artificial Dynamical Systems, Eds M.A. Aziz-Alaoui and C. Bertelle, Understanding complex systems series, Springer, pp 129-147

3. I. Belykh, E. Lange, M. Hasler (2005), Synchronization of Bursting Neurons: What matters in the Network Topology, Phy. Rev. Lett.94, 18, pp 188101.1188101.4

4. S. Boccaletti, J. Kurths, G. Osipov, D. Valladares, C. Zhou (2002), The synchronization of chaotic systems, Physics Reports 366, pp 1-101

5. N. Corson, M.A. Aziz Alaoui (2009), Asymptotic dynamics of the slow-fast Hindmarsh-Rose neuronal system, To appear in DCDIS-B

6. J.M. Gonzalez-Miranda (2007), Complex bifurcation structures in the Hindmarsh-Rose neuron model, International Journal of Bifurcation and Chaos, Vol. 17, 9, pp 3071-3083

7. J.L. Hindmarsh, R.M. Rose (1982), A model of the nerve impulse using two first-order differential equations, Nature, vol. 296, pp 162 - 164

8. J.L. Hindmarsh, R.M. Rose (1984), A model of neuronal bursting using three coupled first order differential equations, Proc. R. Sc. Lond. B221, pp 87-102

9. E. Mosekilde, B. Lading, S. Yanchuk, Y. Maistrenko (2001), Bifurcation structure of a model of bursting pancreatic cells, BioSystems, Vol 63, pp 3-13

10. G. Innocenti, A. Morelli, R. Genesio, A. Torcini (2007), Dynamical phases of the Hindmarsh-Rose neuronal model : Studies of the transition from bursting to spiking chaos, Chaos, Vol 17, 043128, pp 1-11

11. E.M. Izhikevich (2007), Dynamical systems in neuroscience - The geometry of excitability and bursting, The MIT Press

12. L.M. Pecora, T.L. Caroll (1998), Master stability functions for synchronized coupled systems, Phys. Rev. Lett.80, 10, pp 2109-2112 
13. A. Pikovsky, M. Rosenblum, J. Kurths (2001), Synchronization, A universal concept in nonlinear sciences, Cambridge Nonlinear Science Series 12

14. D. Terman (1991), Chaotic spikes arising from a model of bursting in excitable membranes, SIAM J. Appl. Math. 51, 5, pp 1418-1450

15. D.Terman (1992), The transition from bursting to continuous spiking in excitable membrane models, J. Nonlinear Sc. 2, pp 135-182

16. X.J. Wang (1993), Genesis of bursting oscillations in the Hindmarsh-Rose model and homoclinicity to a chaotic saddle, Physica D, Vol 62, Issues 1-4, pp 263-274

17. Z.Q. Yang, Q.S. Lu (2004) Characteristics of Period-Adding Bursting Bifurcation Without Chaos in the Chay Neuron Model, Chin.Phy.Let., Vol 21, Num 11, pp 2124-2128 